\title{
On Nikaidô's proof of the invariant mean-value theorem
}

B. J. PETTIS (Chapel Hill)

Dedicated to Professor S. Mazur and Professor W. Orlicz

The proof [4] mentioned in the title is actually a proof of what might be called "almost a fixed-point theorem", in this case one that asserts that for a semigroup $\Phi$ of continuous affine maps in a compact convex set $K$ there will be, under certain circumstances, some $\bar{\varphi}$ in $\Phi$ and some $\bar{x}$ in $K$ such that $\bar{\varphi}(\varphi(\bar{x}))=\bar{\varphi}(\bar{x})$ for every $\varphi$ in $\Phi$. Such theorems were established earlier by Peck [5] and Klee [3]; after Nikaido, Cohen and Collins [2] were the first to observe that his proof established a theorem of the above sort. Here, using the same proof, we present a slightly more general version, Theorem 0 below [6], from which follow the above theorems as well as some others, including Kakutani's on equicontinuous groups.

Throughout, except in Theorem $0, K$ is a compact convex set in a real linear topological space $E$ that is separated by its dual $E^{*}, K^{\mathcal{K}}$ is the set of all functions on $K$ to $K$ and has the product topology, $\Phi$ is a subsemigroup of $K^{K}$ and has all,its elements affine, and $\Psi$ is the closure of $\Phi$ in $K^{K}$. We recall that under these circumstances $\Psi$ is compact, has its elements affine, and as a set of maps in $K$ has the same fixed points as $\Phi$. If $\Phi$ is equicontinuous, then so is $\Psi$, and hence the elements of $\Psi$ are continuous and affine, $\Psi$ is a subsemigroup of $K^{K}$, and the maps $(\psi, x) \rightarrow \psi(x)$ and $\left(\psi, \psi^{\prime}\right) \rightarrow \psi\left(\psi^{\prime}\right)$ are continuous on $\Psi \times K$ to $K$ and on $\Psi \times \Psi$ to $\Psi$; in particular, $\Psi$ is a compact topological subsemigroup of $K^{K}$.

THEOREM 1. If $\Phi$ is equicontinuous there exist $\bar{\psi}$ in $\Psi$ and $\bar{x}$ in $K$ such that $\bar{\psi}(\bar{x})=\bar{\psi}(\psi(\bar{x}))$ for every $\psi$ in $\Psi$.

From this there follow these fixed point results.

COROLLARY 1. If $\Phi$ is equicontinuous and if given $x$ in $K, \varphi$ and $\varphi^{\prime}$ in $\Phi$, and $U$ a nucleus in $E$ there is some $\varphi^{\prime \prime}$ in $\Phi$ such that $\varphi^{\prime \prime}\left(\varphi^{\prime}(\varphi(x))\right)$ $-\varphi(x) \in U$ and $\varphi^{\prime \prime}\left(\varphi^{\prime}(x)\right)-x \in U$, then $\Psi$ has a fixed point. 
Kakutani's theorem that $\Phi$ has a fixed point if it is an equicontinuous group follows immediately.

COROLLARY 2. If $\Phi$ is equicontinuous and if given $x$ in $K, \varphi$ and $\varphi^{\prime}$ in $\Phi$, and $U$ any nucleus in $E$ there is some $\varphi^{\prime \prime}$ in $\Phi$ such that $\varphi\left(\varphi^{\prime \prime}(x)\right)-$ $-\varphi^{\prime}(\varphi(x)) \in U$, then $\Psi$ has a fixed point.

Corollary 2 is a variant of the Markoff-Kakutani theorem; commutativity has been replaced by equicontinuity plus a weak form of commutativity. It implies I.2.13 of [1].

To obtain these from Theorem 1 we use the following, the proof of which is postponed to the end of this section:

LEMMA. If $\Phi$ satisfies the hypotheses of Corollary 1 or of Corollary 2, then so does $\Psi$.

Thus under the conditions of Corollary 1 we know from Theorem 1 that there are $\bar{x}$ and $\bar{\psi}$ such that $\bar{\psi}(\bar{x})=\bar{\psi}(\psi(\bar{x}))$ for every $\psi$ in $\Psi$, and from the lemma that given any $\psi$ in $\Psi$ and any nucleus $U$ in $E$ there is some $\psi^{\prime \prime}$ in $\Psi$ such that $\psi^{\prime \prime}(\bar{\psi}(\psi(\bar{x})))-\psi(\bar{x}) \in U$ and $\dot{\psi}^{\prime \prime}(\bar{\psi}(\bar{x}))-\bar{x} \epsilon U$. Thus $\psi(\bar{x})-\bar{x} \epsilon-U+U$, and so $\psi(\bar{x})=\bar{x}$ for every $\psi$ in $\Psi$. For Corollary 2 an even simpler proof establishes that $\bar{\psi}(\bar{x})$ is a fixed point for $\Psi$.

Theorem 1 , in view of the remarks preceding it, will clearly result from the following

THEOREM 0. Let $K$ be a compact convex set in a real linear topological space $E$ having in its dual a subset $K^{*}$ that separates points of $K$. Let $\Psi$ be a semigroup of continuous affine maps of $K$ into $K$ and suppose $\Psi$ has a compact topology such that $f(\psi(x))$ is, for each $f$ in $K^{*}$, continuous on $\Psi \times K$. Then there exist $\bar{\psi}$ in $\Psi$ and $\bar{x}$ in $K$ such that $\bar{\psi}(\bar{x})=\bar{\psi}(\psi(\bar{x}))$ for every $\psi$ in $\Psi$.

For each finite set $\gamma$ in $K^{*}$ and each finite set $\delta$ in $\Psi$ let

$A(\gamma, \delta)=\{(\bar{\psi}, \bar{x}): f(\bar{\psi}(\bar{x}))=f(\bar{\psi}(\psi(\bar{x})))$ for every $f$ in $\gamma$ and every $\psi$ in $\delta\}$.

Since $\Psi \times K$ is compact and $K^{*}$ separates $K$, it is enough to show that each $A(\gamma, \delta)$ is closed and non-void. But $A(\gamma, \delta)$ is closed since each $\psi$ is continuous and since $f(\bar{\psi}(\bar{x}))$ is continuous in $(\bar{\psi}, \bar{x})$ for each $f$. To show that it is non-void suppose $\gamma=\left\{f_{1}, \ldots, f_{m}\right\}$ and $\delta=\left\{\psi_{1}, \ldots, \psi_{n}\right\}$. Let

$$
\sigma=\frac{1}{n} \sum_{j=1}^{n} \psi_{j}
$$

then $\sigma$ is continuous and affine in $K$ and hence has a fixed point $\bar{x}$. Define $T$ on $\Psi$ to $R^{m}$ by $T(\psi)=\left(f_{1}(\psi(\bar{x})), \ldots, f_{m}(\psi(\bar{x}))\right)$; clearly, $T$ is continuous.
Since

$$
f_{i}(\psi(\bar{x}))=f_{i}(\psi(\sigma(\bar{x})))=f_{i}\left(\psi\left(\frac{1}{n} \sum_{j=1}^{n} \psi_{j}(\bar{x})\right)\right)=\frac{1}{n} \sum_{j=1}^{n} f_{i}\left(\psi \psi_{j}(\bar{x})\right),
$$

we have

$$
T(\psi)=\frac{1}{n} \sum_{j=1}^{n} T\left(\psi \psi_{j}\right)
$$

The function $\|T(\psi)\|$, being continuous on compact $\Psi$, attains its maximum at some $\bar{\psi}$; then

$$
\|T(\bar{\psi})\|=\left\|\frac{1}{n} \sum_{j=1}^{n} T\left(\bar{\psi} \psi_{j}\right)\right\| \leqslant \frac{1}{n} \sum_{j=1}^{n}\left\|T\left(\bar{\psi} \psi_{j}\right)\right\| \leqslant \frac{1}{n} \sum_{j=1}^{n}\|T(\bar{\psi})\|
$$

and hence $T(\bar{\psi})=T\left(\bar{\psi} \psi_{j}\right)$ for $j=1, \ldots, n$. Thus $f_{i}(\bar{\psi}(\bar{x}))=f_{i}\left(\bar{\psi} \psi_{j}(\bar{x})\right)$ for all $i, j$ and so $A(\gamma, \delta)$ is not void.

Reverting to the proof of the lemma, suppose $\Phi$ satisfies the hypotheses of Corollary 1. Then $\Psi$ is equicontinuous; and given $x$ in $K, \psi$ and $\psi^{\prime}$ in $\Psi$, and any nucleus $U$ in $E$, some $\psi^{\prime \prime}$ in $\Psi$ must be found such that $\psi^{\prime \prime}\left(\psi^{\prime}(\psi(x))\right)-\psi(x) \in U$ and $\psi^{\prime \prime}\left(\psi^{\prime}(x)\right)-x \epsilon U$. We may suppose $U$ to be closed. Choose nets $\left\{\varphi_{a}\right\}$ and $\left\{\varphi_{a}^{\prime}\right\}$ in $\Phi$ converging to $\psi$ and $\psi^{\prime}$ in $K^{K}$. From the assumptions on $\Phi$ there is for each a some $\varphi_{a}^{\prime \prime}$ in $\Phi$ such that $\varphi_{a}^{\prime \prime}\left(\varphi_{a}^{\prime}\left(\varphi_{a}(x)\right)\right)-\varphi_{a}(x) \epsilon U$ and $\varphi_{a}^{\prime \prime}\left(\varphi_{\alpha}^{\prime}(x)\right)-x \epsilon U$. Since $\Psi$ is compact, the net $\left\{\varphi_{a}^{\prime \prime}\right\}$ clusters at some $\psi^{\prime \prime}$ in $\Psi$; we may presume that. $\left\{\varphi_{\alpha}^{\prime \prime}\right\}$ converges to $\psi^{\prime \prime}$. From the remarks preceding. Theorem 1, $\Psi$ is a topological semigroup; hence $\varphi_{a}^{\prime \prime}\left(\varphi_{a}^{\prime}\left(\varphi_{a}\right)\right)$ converges to $\psi^{\prime \prime}\left(\psi^{\prime}(\psi)\right)$ and $\varphi_{a}^{\prime \prime}\left(\varphi_{a}^{\prime}\right)$ to $\psi^{\prime \prime}\left(\psi^{\prime}\right)$ in $K^{K}$; thus $\varphi_{\alpha}^{\prime \prime}\left(\varphi^{\prime}\left(\varphi_{\alpha}(x)\right)\right)$ converges to $\psi^{\prime \prime}\left(\psi^{\prime}(\psi(x))\right)$ and $\varphi_{a}^{\prime \prime}\left(\varphi_{a}^{\prime}(x)\right)$ to $\psi^{\prime \prime}\left(\psi^{\prime}(x)\right)$ in $K$, so that $\varphi_{\alpha}^{\prime \prime}\left(\varphi_{a}^{\prime}\left(\varphi_{\alpha}(x)\right)\right)-\varphi_{a}(x)$ converges to $\psi^{\prime \prime}\left(\psi^{\prime}(\psi(x))\right)-$ $-\psi(x)$ and $\varphi_{a}^{\prime \prime}\left(\varphi_{a}^{\prime}(x)\right)-x$ to $\psi^{\prime \prime}\left(\psi^{\prime}(x)\right)-x$. Since $U$ is closed, we have $\psi^{\prime \prime}\left(\psi^{\prime}(\psi(x))\right)-\psi(x)$ in $U$ and $\psi^{\prime \prime}\left(\psi^{\prime}(x)\right)-x$ in $U$, as desired.

A similar proof covers the case of Corollary 2.

From Theorem 0 there also follow immediately earlier theorems due to Peck [5] and Klee $(4.3,4.4$, and 3.1 (d) of [3]) and the Cohen and Collins theorem (Theorem 2 of [2], and II.3.14 of [1]).

For other applications let $S$ be a set and $\Phi$ a semigroup of transformations in $S$. Let $E$ be a linear topological space of functions on $S$ and for each $x$ in $E$ and each $\varphi$ in $\Phi$ let $T_{\varphi}(x)=x(\varphi)$. Suppose $K$ is a compact convex set in $E$ such that $T_{\varphi}(K) \subset K$ for every $\varphi$ in $\Phi$. If there is a compact topology for $\mathscr{T}=\left\{T_{\varphi}: \varphi\right.$ in $\left.\Phi\right\}$ such that $(T, x) \rightarrow T(x)$ is continuous on 
$T \times K$ to $K$, then there are $\bar{x}$ in $K$ and $\bar{\varphi}$ in $\Phi$ such that $\bar{x}(\bar{\varphi}(s))=\bar{x}(\varphi(\bar{\varphi}(s)))$ for every $\varphi$ in $\Phi$ and every $s$ in $S$. If, moreover, given any $s, s^{\prime}$ in $S$ there are $\varphi, \varphi^{\prime}$ in $\Phi$ such that $\varphi(s)=\varphi^{\prime}\left(s^{\prime}\right)$, then $\bar{x}(\bar{\varphi})$ is a constant element of $K$.

More particularly, if $S$ is compact regular and $\Phi$ is a semigroup of transformations in $S$ such that $\Phi$ has a compact regular topology such that $(\varphi, s) \rightarrow \varphi(s)$ is continuous, and if $F$ is a complete locally convex linear topological space and $E$ is the space of continuous functions on $S$ to $F$ topologized by uniform convergence, let $O_{x}=\left\{x(\varphi): \varphi\right.$ in $\left.\Phi_{\}}\right\}$for each $x$ in $E$ and $K_{x}=$ the closed convex eover of $O_{x}$. Then for each $x$ in $E$ there exists $y_{x}$ in $K_{x}$ and $\varphi_{x}$ in $\Phi$ such that $y_{x}\left(\varphi_{x}(s)\right)=y_{x}\left(\varphi\left(\varphi_{x}(s)\right)\right)$ for all $\varphi$ and $s$; if, moreover, given $s, s^{\prime}$ there are $\varphi, \varphi^{\prime}$ such that $\varphi(s)$ $=\varphi^{\prime}\left(s^{\prime}\right)$, then for each $x$ the function $y_{x}$ is constant. For, the map $(\varphi, s) \rightarrow \varphi(s)$ is uniformly continuous and hence the map $(x, \varphi) \rightarrow x(\varphi)$ is continuous on $E \times \Phi$ to $E$. Clearly $O_{x}$ is then compact for each $x$ and so therefore is $K_{x}$ since $E$ is complete. Applying the preceding paragraph to $K_{x}$ for each $x$ yields the above conclusion [4].

\section{References}

[1] J. F. Berglund and K. H. Hofmann, Compact semitopological semigroups and weakly almost periodic functions, Berlin 1967.

[2] H. Cohen and H. S. Collins, Affine semigroups, Trans. Amer. Math. Soc. 93 (1959), p. 97-113.

[3] V. L. Klee, Jr., Invariant extension of linear functionals, Pac. Jour. of Math. 4 (1954), p. 37-46.

[4] H. Nikaidô, A proof of the invariant mean-value theorem on almost periodic functions, Proc. Amer. Math. Soc. 6 (1955), p. 361-363.

[5] J. E. L. Peck, An ergodic theorem for a non-commutative semigroup of linear operators, ibidem 2 (1951), p. 414-421.

[6] B. J. Pettis, Abstract, Notices Amer. Math. Soc. 6 (1959), p. 282.

THE UNIVERSITY OF NORTH CAROLINA, CHAPEL HILL

Reçu par la Rédaction le 5. \%. 1968

\section{On equations with reflection}

by

If an equation contains together with the unknown function $x(t)$ the value $x(-t)$, then it will be called an equation with reflection. For example, the differential equation

$$
a_{0} x(t)+b_{0} x(-t)+a_{1} x^{\prime}(t)+b_{1} x^{\prime}(-t)=y(t)
$$

is an equation with reflection.

Let us denote the reflection by $S$. Since $S^{2}=I$, where $I$ is identity operator, $S$ is an involution. The differentiation operator $D$ is anticommuting with $S$. Indeed,

$$
(S D x)(t)=x^{\prime}(-t), \quad(D S x)(t)=x(-t)^{\prime}=-x^{\prime}(-t)=(-S D x)(t) .
$$

Hence $S D+D S=0$.

In this paper we shall consider a linear equation

$$
\left(a_{0} I+b_{0} S\right) x+\left(a_{1} I+b_{1} S\right) D x=y
$$

where $S$ is an involution on a linear space $X, D$ is a linear operator acting in $X$ and anticommuting with $S$, and $a_{0}, b_{0}, a_{1}, b_{1}$ are scalars.

As examples we shall consider equation (1) and an integral equation of form (2).

1. Let $X$ be a linear space (over complex scalars). Let $S$ be an involution: $S^{2}=I$ on $X$. Let

$$
P^{+}=\frac{1}{2}(I+S), \quad P^{-}=\frac{1}{2}\left(I-S^{\prime}\right) .
$$

The following properties of an involution, shown in [1] (see also [2]) will be used further:

$1^{\circ}$ The operators $P^{+}$and $P^{-}$are disjoint projectors giving a partition of unity:

(1.1) $P^{+} P^{-}=P^{-} P^{+}=0, \quad\left(P^{+}\right)^{2}=P^{+}, \quad\left(P^{-}\right)^{2}=P^{-}, \quad P^{+}+P^{-}=I$

Moreover, $P^{+}-P^{-}=S, S P^{+}=P^{+}, S P^{-}=-P^{-}$. 